\title{
AN ADAPTIVE ALGORITHM FOR RESTORING IMAGE CORRUPTED BY MIXED NOISE
}

\author{
Pham Cong Thang * \\ The University of Danang, \\ University of Science and Technology \\ Danang, Viet Nam \\ pcthang@dut.udn.vn
}

\author{
Tran Thi Thu Thao \\ The University of Danang, \\ University of Economics \\ Danang, Viet Nam \\ thaotran@due.udn.vn
}

\author{
Phan Tran Dang Khoa \\ The University of Danang, \\ University of Science and Technology \\ Danang, Viet Nam \\ ptdkhoa@dut.udn.vn
}

\author{
Dinh Viet Sang \\ Hanoi University of Science \\ and Technology \\ Hanoi, Viet Nam \\ sangdv@soict.hust.edu.vn
}

\author{
Pham Minh Tuan \\ The University of Danang, \\ University of Science and Technology \\ Danang, Viet Nam \\ pmtuan@dut.udn.vn
}

\author{
Nguyen Minh Hieu \\ The University of Danang, \\ University of Science and Technology \\ Danang, Viet Nam \\ nmhieu@dut.udn.vn
}

Article history:

Received 10.09.2019, Accepted 28.09.2019

\begin{abstract}
Image denoising is one of the fundamental problems in image processing. Digital images are often contaminated by noise due to the image acquisition process under poor conditions. In this paper, we propose an effective approach to remove mixed Poisson-Gaussian noise in digital images. Particularly, we propose to use a spatially adaptive total variation regularization term in order to enhance the ability of edge preservation. We also propose an instance of the alternating direction algorithm to solve the proposed denoising model as an optimization problem. The experiments on popular natural images demonstrate that our approach achieves superior accuracy than other recent state-of-the-art techniques.
\end{abstract}

\section{Key words}

Image denoising, total variation, adaptive regularization.

\section{Introduction}

Image degradation is the result of defects of the imaging system and noise coming from the formation, transmission and recording processes. Let $\Omega \subset \mathbb{R}^{2}$ be a bounded open set, and let $u(x): \Omega \rightarrow \mathbb{R}$ be a true image describing a real scene, and let $f(x)$ be the observed image of the same scene $\left(x=\left(x_{1}, x_{2}\right) \in \Omega\right)$, which is a degraded image of $u$. In general, image restoration is often formulated as the problem of reconstructing a true image $u$ with the size of $(M \times N)$ corrupted by random noise $\eta$, from an observed image $f$. The sought-for image $u$ is a solution of the corresponding inverse problem [Pham, 2015; Pham, 2018].

A number of algorithms, some of which are based on total variation (TV) regularization, have been proposed for solving the denoising problem. One of successful edge preserving image denoising models is the wellknown ROF model [Rudin, 1992]. The ROF model is defined by the following unconstrained discrete minimization problem:

$$
\min _{u}\left(\|u\|_{T V}+\frac{\lambda}{2}\|u-f\|_{2}^{2}\right)
$$

where the first term stands for the total variation of $u$ corresponding to the image prior, and the second term is the data fidelity term measuring the error between the true and observed images; $\lambda$ is a positive regularization parameter, $\|\cdot\|_{T V}$ is the total variation regularization term given later, cf. Eq. (8).

Recently, the authors in [Huang, 2008] introduced a auxiliary variable $z$ and proposed a fast total variation minimization method to solve problem (1) as follows:

$$
\min _{u, z}\left(\|z\|_{T V}+\frac{\gamma}{2}\|u-z\|_{2}^{2}+\frac{\lambda}{2}\|u-f\|_{2}^{2}\right)
$$

The ROF models (1) and (2) are appropriate to remove additive Gaussian noise. However, many imaging devices, such as digital cameras, TEP and SPECT tomography, measure scene irradiance by counting the number

${ }^{*}$ Corresponding author 
of photons incoming on the sensor. Each photon detection can be considered as an independent event, leading to the photon noise. The uncertainty of photon counting can be modeled using a Poisson noise distribution, for which the variance of noise that corrupts the signal depends on the value of that signal. To remove Poisson noise, Le et al. [Le, 2007] proposed a denoising model as follows:

$$
\min _{u}\left(\|u\|_{T V}+\beta\langle 1, u-f \log u\rangle\right),
$$

where $\beta$ is a regularization parameter; $u$ must be positive almost everywhere over $\Omega$.

Compared to the ROF model, the regularization parameter of the functional described in (3) depends on the reconstructed image $u$, that better suits for Poisson noise, which increases with image intensity. To better improve the edge-preserving removal of Poisson noise, the authors in [Zhou, 2012 ] proposed an adaptive model of (3) described as follows (M1):

$$
\min _{u}\left(\alpha(x)\|u\|_{T V}+\beta\langle 1, u-f \log u\rangle\right),
$$

where $\alpha(x)$ is an edge-detection function given later, cf. Eq. (7).

As suggested in [Calatroni, 2017; Reyes, 2013], Eq. (1) and (3) can be combined to denoise an image corrupted by mixed Poisson-Gaussian noise (M2):

$$
\begin{aligned}
\min _{u}\left(\|u\|_{T V} \|\right. & +\frac{\lambda_{1}}{2}\|u-f\|_{2}^{2} \\
& \left.+\lambda_{2}\langle 1, u-f \log u\rangle\right) .
\end{aligned}
$$

where $\lambda_{1}$ and $\lambda_{2}$ are positive regularization parameters; $u$ must be positive almost everywhere over $\Omega$

Inspired by models (2), (4) and (5), we propose the following unconstrained minimization problem to denoise an image corrupted by mixed Poisson-Gaussian noise (M3) :

$$
\begin{aligned}
\min _{u}\left(\alpha(x)\|z\|_{T V}\right. & +\frac{\gamma}{2}\|u-z\|_{2}^{2}+\frac{\lambda_{1}}{2}\|u-f\|_{2}^{2} \\
& \left.+\lambda_{2}\langle 1, u-f \log u\rangle\right) .
\end{aligned}
$$

where $u$ must be positive almost everywhere over $\Omega ; \lambda_{1}$ and $\lambda_{2}$ are positive regularization parameters, $\alpha(x)$ is an edge detection function defined in (7).

Overall the years, many efficient methods have been proposed, for instance, gradient descent method [Rudin, 1992; Wang, 2011], Chambolle's projection algorithm [Chambolle, 2004], Split Bregman method [Goldstein, 2009], alternating direction algorithms[Huang, 2008; $\mathrm{He}, 2014]$, which can be used to obtain the solution of the resulting convex optimization problem (6).

In this paper, we study an effective method for image restoration corrupted by mixed Poisson-Gaussian noise.
We propose to use a spatially adaptive total variation regularization term in order to enhance the ability of edge preservation. To solve the energy minimization problem (6), we employ an alternating direction algorithm which is highly efficient in terms of computational time.

The remaining of the paper is organized as follows: in Section (2), which is the main of our contributions, we discuss the proposed model and numerical method to solve the minimization problem. Section (3) consists of experiments and discussions. Finally, conclusions are made in Section (4).

\section{The Proposed Approach}

In this paper, our objective is to solve the optimization problem (6):

$$
\begin{aligned}
\min _{u, z}\left(\alpha(x)\|z\|_{T V}\right. & +\frac{\gamma}{2}\|u-z\|_{2}^{2}+\frac{\lambda_{1}}{2}\|u-f\|_{2}^{2} \\
& \left.+\lambda_{2}\langle 1, u-f \log u\rangle\right) .
\end{aligned}
$$

The function $\alpha(x)$ can be chosen typically as follows [Catte, 1992]:

$$
\alpha(x)=\frac{1}{1+\frac{|v(x)|}{K}},
$$

where $v(x)=\left(\left|\nabla G_{\sigma}(x) * f\right|\right)^{2}, K$ is a threshold value, operator $*$ denotes the convolution,

$G_{\sigma}(x)=\frac{1}{2 \pi \sigma^{2}} \exp \left(-\frac{x^{2}}{2 \sigma^{2}}\right)$ - stands for the Gaussian filter with standard deviation $\sigma$.

We can write $u_{i, j}$ for the pixel at coordinates $(i, j)$ in image $u(i=1, . ., M ; j=1, . ., N)$. The operator $\|\nabla u\|_{T V}$ is defined as follows:

$$
\begin{aligned}
& \nabla u_{i, j}=\left(\nabla_{1} u(i, j), \nabla_{2} u(i, j)\right), \\
& \nabla_{1} u(i, j)=u(i+1, j)-u(i-1, j), \\
& \nabla_{2} u(i, j)=u(i, j+1)-u(i, j-1), \\
& \|u\|_{T V}=\sum_{j, k}\|\nabla u(i, j)\|_{2} \\
& =\sqrt{\left|\nabla_{1} u(i, j)\right|^{2}+\left|\nabla_{2} u(i, j)\right|^{2}+\varepsilon^{2}} .
\end{aligned}
$$

where $\varepsilon$ is a small positive quantity, added for considerations of numerical stability.

We have two decoupled variables $u, z$ in (6). The alternating minimization method to solve problem (6) can be expressed as follows. Given initial values $u^{(0)}$ and $z^{(0)}$, solving the unconstrained problem (6) is performed via the following iterative scheme:

$$
\begin{aligned}
& u^{(k+1)}=\underset{u}{\arg \min }\left(\frac{\gamma}{2}\left\|u-z^{(k)}\right\|^{2}+\right. \\
& \left.\frac{\lambda_{1}}{2}\|u-f\|^{2}+\lambda_{2}\langle 1, u-f \log u\rangle\right),
\end{aligned}
$$




$$
\begin{array}{r}
z^{(k+1)}=\underset{z}{\arg \min }\left(\alpha(x)\|z\|_{T V}\right. \\
\left.+\frac{\gamma}{2}\left\|u^{(k)}-z\right\|^{2}\right) .
\end{array}
$$

In fact, the problem (6) is constructed by making the substitutions $u$ by $z$ in (5). Here, we add an auxiliary variable $b$ on the update of variables $u$ and $z$. The auxiliary variable $b$ controls the residual between $u$ and $z$, improving the convergence and stability of the solution. This yields a new scheme described as follows:

$$
\begin{gathered}
u^{(k+1)}=\underset{u}{\arg \min }\left(\frac{\gamma}{2}\left\|u-z^{(k)}-b^{(k)}\right\|^{2}+\right. \\
\left.\frac{\lambda_{1}}{2}\|u-f\|^{2}+\lambda_{2}\langle 1, u-f \log u\rangle\right), \\
z^{(k+1)}=\underset{z}{\arg \min }\left(\alpha(x)\|z\|_{T V}\right. \\
\left.+\frac{\gamma}{2}\left\|u^{(k)}-z-b^{(k)}\right\|^{2}\right), \\
b^{(k+1)}=b^{(k)}+\left(z^{(k+1)}-u^{(k+1)}\right),
\end{gathered}
$$

where $k=0,1,2, \ldots$ is iteration number.

The $u$ subproblem (9) is a quadratic optimization problem. Therefore, we have the following optimality condition:

$$
\gamma\left(u-z^{(k)}-b^{(k)}\right)+\lambda_{1}(u-f)+\lambda_{2}\left(1-\frac{f}{u}\right)=0 .
$$

Multiplying both sides of this equation by $u$, we get:

$$
\begin{aligned}
& \left(\gamma+\lambda_{1}\right) u^{2} \\
& -\left(\gamma z^{(k)}+\gamma b^{(k)}+\lambda_{1} f-\lambda_{2}\right) u-\lambda_{2} f=0 .
\end{aligned}
$$

The solution $u$ of the equation (9) is a positive solution of Eq. (12) given by:

$$
u^{(k+1)}=q^{(k)}+\sqrt{\left(q^{(k)}\right)^{2}+\frac{\lambda_{2} f}{\lambda_{1}+\gamma}},
$$

where

$$
q^{(k)}=\frac{\gamma z^{(k)}+\gamma b^{(k)}+\lambda_{1} f-\lambda_{2}}{2\left(\lambda_{1}+\gamma\right)} .
$$

Clearly, the problem (10) can be solved by different TV denoising methods. In this work, we employ the Chambolles projection algorithm [Chambolle, 2004] to solve the $z$ subproblem (see Algorithm 1).
Algorithm 1: Adaptive Chambolles projection algorithm for solving Eq. (10)

1. Initialize: $u^{(k+1)}, b^{(k)}, k=0, p^{(0)}=0$

2. while $\left(\left\|p^{(k+1)}-p^{(k)}\right\|_{2}>\varsigma\right)$ do

3. for all values at coordinates $(i, j)$ do

4.

$p_{(i, j)}^{(k+1)}=\frac{p_{(i, j)}^{(k)}+\Delta t\left(\nabla\left(\operatorname{div}\left(\alpha(x) p^{(k)}\right)-\gamma\left(u^{(k+1)}+b^{(k)}\right)\right)\right)_{(i, j)}}{1+\Delta t\left|\nabla\left(\operatorname{div}\left(\alpha(x) p^{(k)}\right)-\gamma\left(u^{(k+1)}+b^{(k)}\right)\right)_{(i, j)}\right|}$

5. end for

6. $k=k+1$

7. end while

8. return $z^{(k+1)}=u^{(k+1)}+b^{(k)}-\frac{1}{\gamma} \operatorname{div}\left(p^{(k+1)}\right)$

The operator $\operatorname{div}\left(p^{(k+1)}\right)$ in Algorithm 1 is defined as follows [Chambolle, 2004]:

$\operatorname{div}(p)_{i, j}=p_{1}(i, j)-p_{1}(i-1, j)+p_{2}(i, j)-p_{2}(i, j-1)$,

where $p_{1}(i, j), p_{2}(i, j)$ is the dual variable at the $(i, j)$ pixel location, $p_{1}(0, j), p_{2}(i, 0)=0$.

Finally, we update the auxiliary variable $b$ by (11) :

$$
b^{(k+1)}=b^{(k)}+\left(z^{(k+1)}-u^{(k+1)}\right) .
$$

The resulting image denoising algorithm is described in Algorithm 2.

Algorithm 2: Adaptive Algorithm for solving the proposed model (6)

1. Initialize: $u^{(0)}=z^{(0)}=f ; b^{(0)}=0 ; k=0$

2. while $\left.\left\|u^{(k+1)}-u^{(k)}\right\|_{2}>\varsigma\right)$ do

3. Compute $u^{(k+1)}$ according to (13)

4. Compute $z^{(k+1)}$ according to Algorithm 1

5. Update $b^{(k+1)}$ according to (14)

6. $k=k+1$

7. end while

8. return $u^{*}=u^{(k+1)}$ 


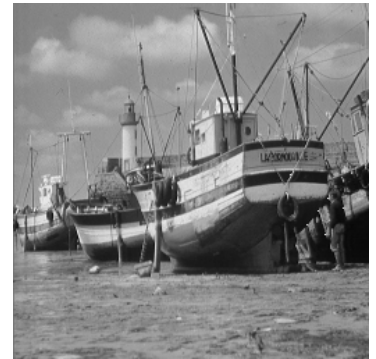

(a) Boat

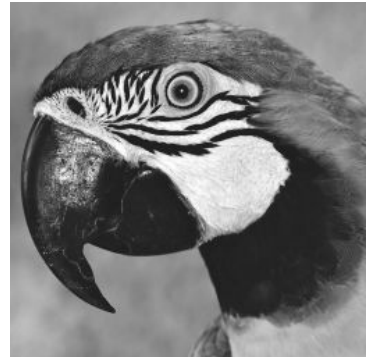

(b) Parrot

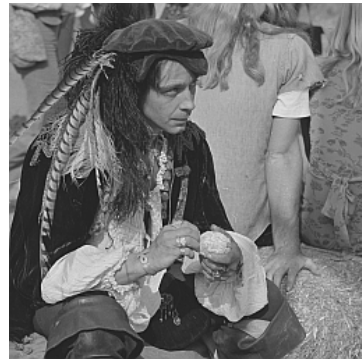

(c) Man

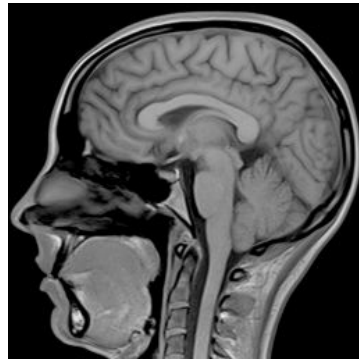

(d) Brain

Figure 1. Standard test images

\section{Experimental results}

In this section, we show some numerical reconstructions obtained by applying our proposed method to mixed Poisson-Gaussian noise. For illustrations, we use the gray level images with size $256 \times 256$ : Boat, Parrot, Man, Brain. The original images are presented in Fig. (1).

The Peak Signal-to-Noise Ratio (PSNR) and Structure Similarity Index (SSIM) [Bovik, 2006] used in comparison are defined as:

$$
\begin{gathered}
P S N R=10 \log _{10}\left(\frac{M N I_{\max }^{2}}{\left\|u^{*}-u\right\|_{2}^{2}}\right), \\
\operatorname{SSIM}\left(u, u^{*}\right)=\frac{\left(2 \mu_{u} \mu_{u^{*}}+c_{1}\right)\left(2 \sigma_{u, u^{*}}+c_{2}\right)}{\left(\mu_{u}^{2}+\mu_{u^{*}}^{2}+c_{1}\right)\left(\sigma_{u}^{2}+\sigma_{u^{*}}^{2}+c_{2}\right)}
\end{gathered}
$$

where $u, u^{*}$ are the original image, the reconstructed or noisy image accordingly; $I_{\max }$ is the maximum intensity of the original image; $M$ and $N$ are the number of image pixels in rows and columns; $\mu_{u}, \mu_{u^{*}}$ - means of images; $\sigma_{u}, \sigma_{u^{*}}$ standard deviations (the square root of variance) of images; $\sigma_{u, u^{*}}$ covariance of two images $u$ and $u^{*} ; c_{1}=\left(K_{1} L\right)^{2}, c_{2}=\left(K_{2} L\right)^{2}, \mathrm{~L}$ is the dynamic range of the pixel values (255 for 8-bit grayscale images), and $K_{1} \ll 1, K_{2} \ll 1$ are small constants.

We show the performance of our proposed method for restoring images contaminated with mixed PoissonGaussian noise. Noisy observations are generated by Poisson noise with some fixed peak $I_{\max }$, and by Gaussian noise with standard deviation $\sigma_{G}$ to the test images (see [Pham, 2018] for more details).

We compare reconstructions using our model with other results using model M1 [Zhou, 2012 ] and model M2 [Calatroni, 2017; Pham, 2018]. For our model, we perform experiments with two cases: the model (6) with constant function $\alpha(x)=1$ and the model (6) with function $\alpha(x)$ given by (7). For simplicity, we name (6) with $\alpha(x)=1$ : model M3; and we name (6) with $\alpha(x)$ given by (7): model $\alpha$-M3.

Meanwhile, all algorithms are implemented using MATLAB on a HP laptop with Intel(R) Core(TM) i7CPU $2.0 \mathrm{GHz}$ and $8 \mathrm{~GB}$ of RAM, Windows 10 (64 bit). For our experiments, we set tolerance $\varsigma=10^{-5}$.
For a fair comparison, we set the regularization parameters of compared methods with their optimal values: $\lambda=0.2, \lambda_{2}=\beta=0.8$. We set the threshold value in (7) $K=10$.

In Fig. (2), we show the denoising results using our models M3 and $\alpha$-M3 for noise level $I_{\max }=120$ and $\sigma_{G}=10$. In Fig. (3), we show the denoising results noise level $I_{\max }=60$ and $\sigma_{G}=5$. As shown in Fig.(2) and Fig. (3), our model $\alpha$-M3 is highly effective for restoring piecewise constant images. This shows that using the proposed model (6) with $\alpha$ function yields better denoising results.

In Fig. (4), we show the denoising results using the compared models for the noise level $I_{\max }=90$ and $\sigma_{G}=10$. Particularly, the first row represents the noisy image, in the others we show respectively the reconstructions using model M1, model M2, our models: M3 and $\alpha$-M3.

An important factor to measure the effectiveness of the denoising methods is run time. Table (1) shows the computational time (in seconds) in case of mixed noise $I_{\max }=90, \sigma_{G}=10$ (Fig. 4). It can be observed from the table that the computation time of the restored images using our models and model M1 is about the same. The cost time of the restored images using our models is shorter than those of the model M2. Fig. (5) shows that the restored pixel intensity curves from the proposed models actually provide a better approximation to the smooth fragments of original pixel intensity curves than those from the other models.

Table 1. Computational time (in seconds) results of the compared methods on test images with $I_{\max }=90, \sigma_{G}=10$

\begin{tabular}{|l|l|l|l|l|}
\hline Image & Model M1 & Model M2 & Model M3 & Model $\alpha$-M3 \\
\hline Boat & 0.5063 & 1.9605 & 0.4820 & 0.4967 \\
\hline Parrot & 0.5084 & 2.1860 & 0.4932 & 0.5138 \\
\hline Man & 0.5032 & 2.0943 & 0.4879 & 0.4835 \\
\hline Brain & 0.5490 & 2.0544 & 0.5720 & 0.5284 \\
\hline
\end{tabular}

For the comparison of the performance quantitatively, in Table (2) and Table (3), we report the values of the PSNR, SSIM for the noisy and recovered images. 


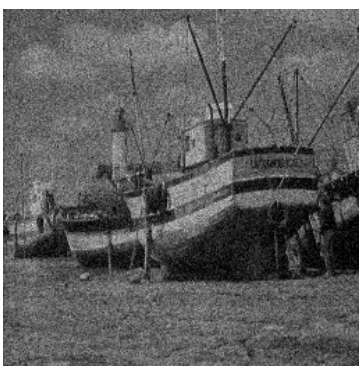

(a)

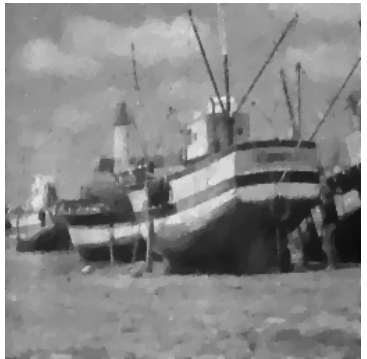

(e)

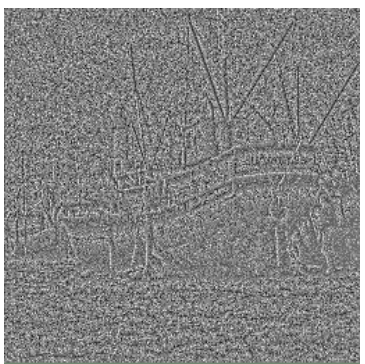

(i)

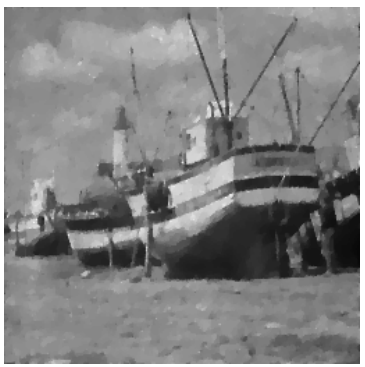

(m)

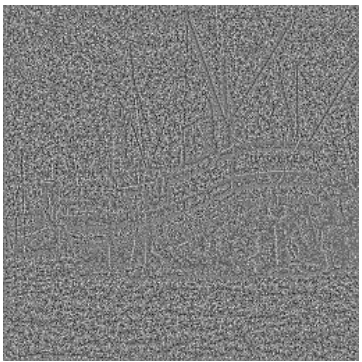

(q)

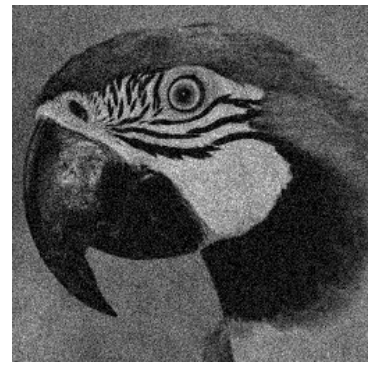

(b)

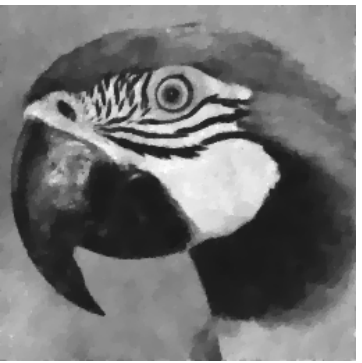

(f)

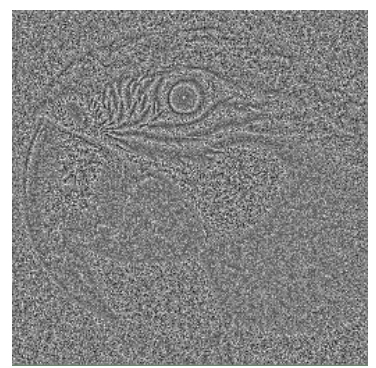

(j)

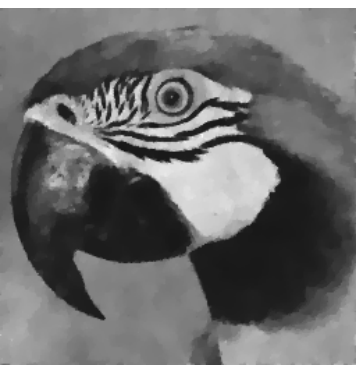

(n)

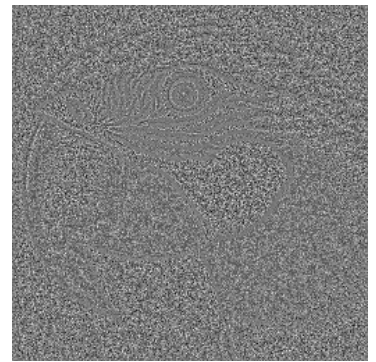

(r)

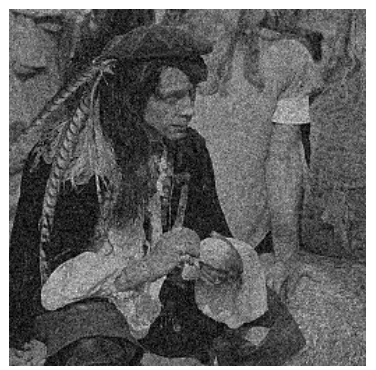

(c)

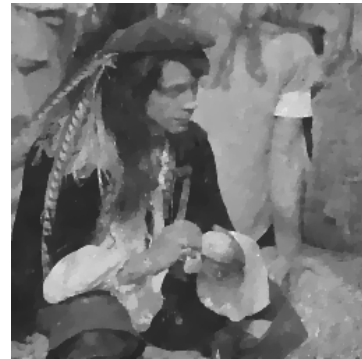

(g)

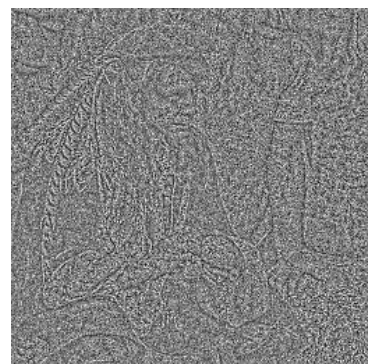

(k)

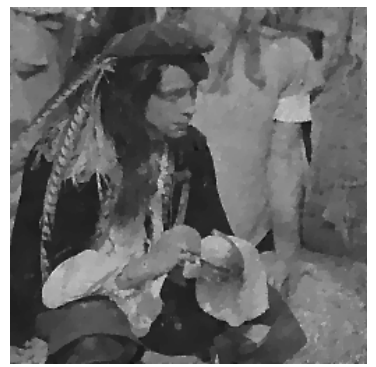

(o)

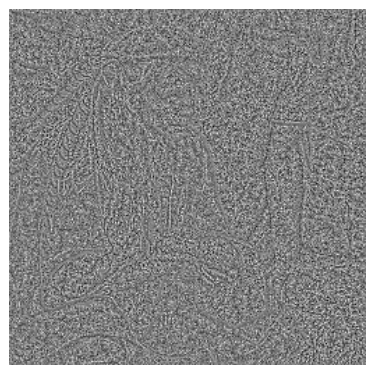

(s)

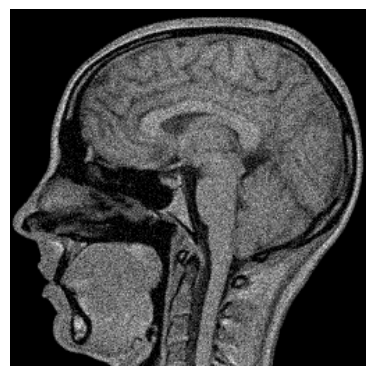

(d)

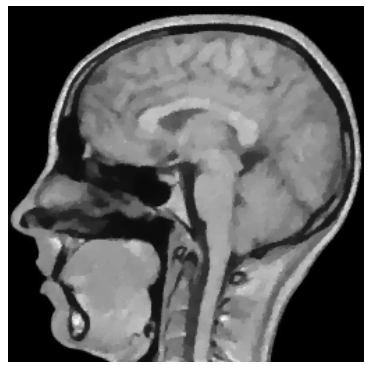

(h)

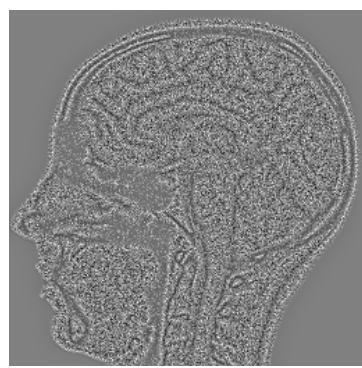

(1)

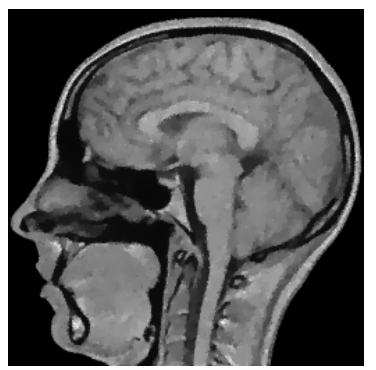

(p)

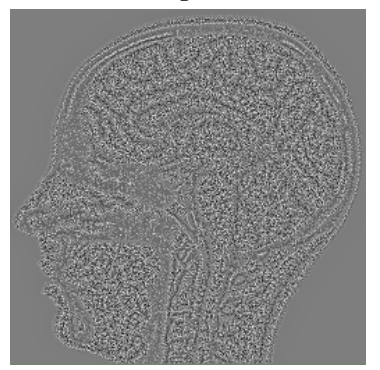

(t)

Figure 2. Recovered results. First row (a - d): Noisy image $f$ with $I_{\max }=120, \sigma=10$; Second row (e - h): restored images using model $\mathbf{M 3}(\gamma=12)$; Third row (i - 1): $f-u$ with our model M3; Fourth row (m-p): restored images using model $\alpha$-M3 $(\gamma=12)$; Fifth row (q t): $f-u$ with our model $\alpha$-M3 


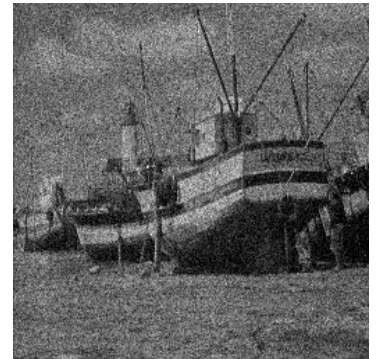

(a)

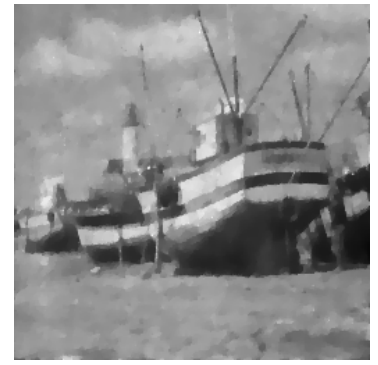

(e)

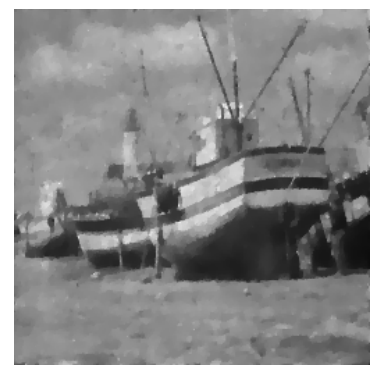

(i)

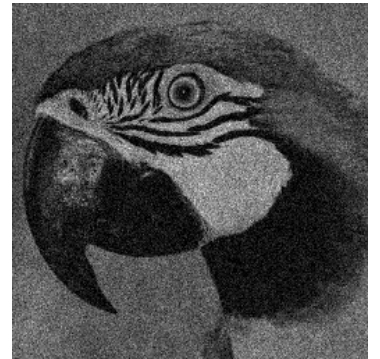

(b)

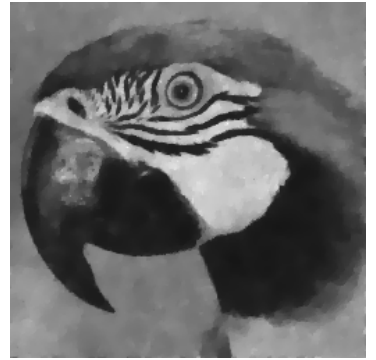

(f)

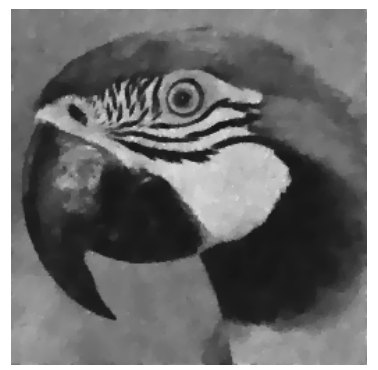

(j)

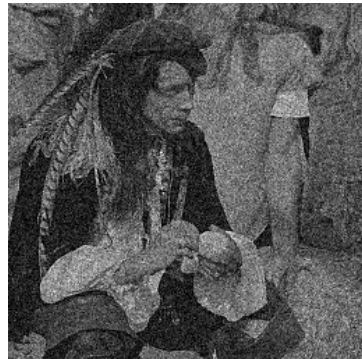

(c)

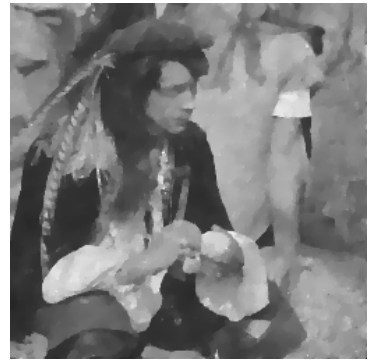

(g)

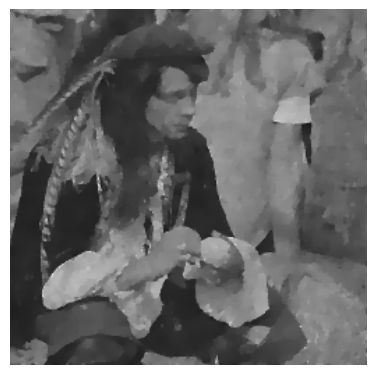

(k)

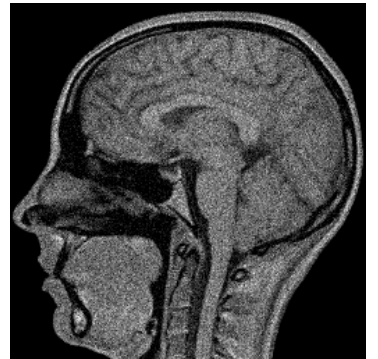

(d)

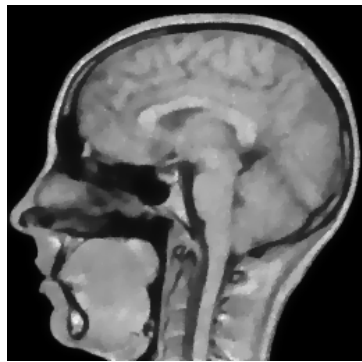

(h)

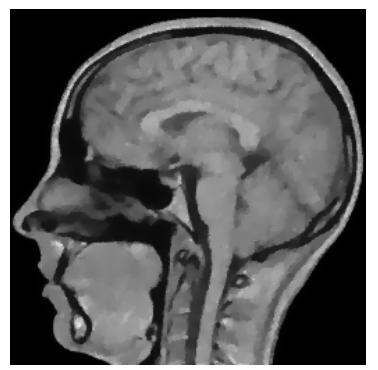

(1)

Figure 3. Recovered results. First row (a - d): Noisy image $f$ with $I_{\max }=60, \sigma=5$; Second row (e - h): restored images with model M1 $(\gamma=8)$; Third column (i - 1): restored images using our model $\alpha-\mathbf{M 3}(\gamma=8)$

As shown in Table (2) and Table (3), The PSNR and SSIM results using our model M3 are better than the results of the model M1. The PSNR and SSIM results using our model $\mathbf{M} 3$ and the model $\mathbf{M} 2$ are about the same. However, the PSNR and SSIM results using our model $\alpha$-M3 are better than the other methods. Thus, we can clearly see that our method outperforms the other relative methods for mixed Poisson-Gaussian noise removal.

\section{Conclusion}

This paper proposes an instance of the alternating minimization method to solve the image denoising problem which is formulated as an unconstrained optimization task with an adaptive total variation smoothing term. Our approach automatically reduces the weight of total variation term near an edge so that it makes the edges less affected by the smoothing term, and, hence, better preserved. The experiments show that our methods yields better results in mixed Poison-Gaussian removal in comparison to other relative methods.

\section{Acknowledgment}

The authors would like to thank the anonymous referees for their helpful remarks and suggestions. This re- search is funded by Funds for Science and Technology Development of The University of Danang under project number B2019-DN02-62.

\section{References}

Benvenuto, F., et al. (2008). The study of an iterative method for the reconstruction of images corrupted by Poisson and Gaussian noise. Inverse Probl., 24(3), 035016.

Bertero, M., Boccacci, P., Desider, G., Vicidomini, G. (2009). Image deblurring with Poisson data: from cells to galaxies. Inverse Probl., 25 (12), 123006.

Bovik, A.C., Wang, Z. (2006). Modern Image Quality Assessment, Synthesis Lectures on Image, Video, and Multimedia Processing. Morgan and Claypool Publishers, 156 pages.

Calatroni, L., De Los Reyes, J. C., Schnlieb, C. B. (2017). Infimal convolution of data discrepancies for mixed noise removal. SIAM. J. Imaging Sci., 10(3), pp. 11961233.

Catte, F., Lions, P. L., Morel, J. M., Coll, T. (1992). Image selective smoothing and edge detection by non- 


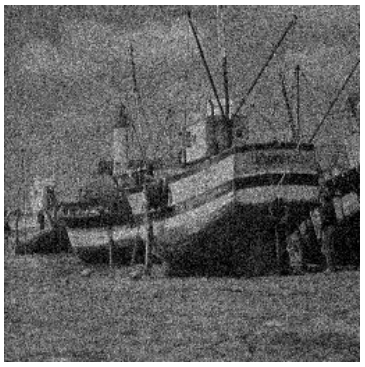

(a)

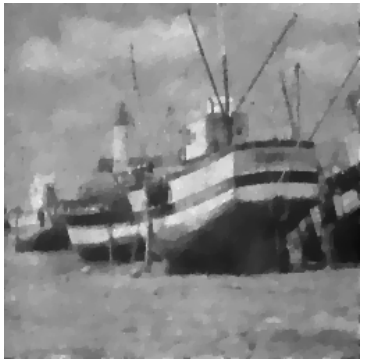

(e)

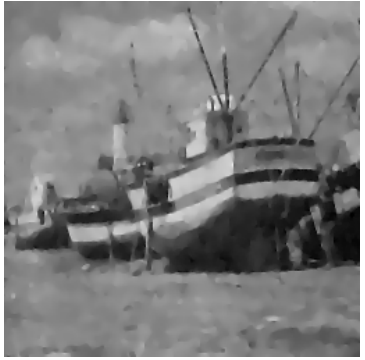

(i)

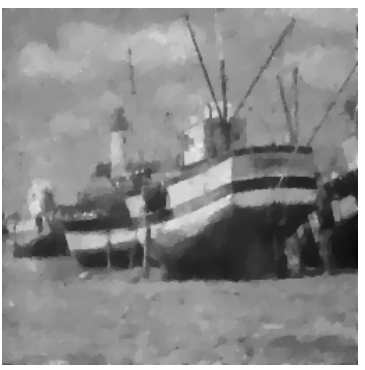

(m)

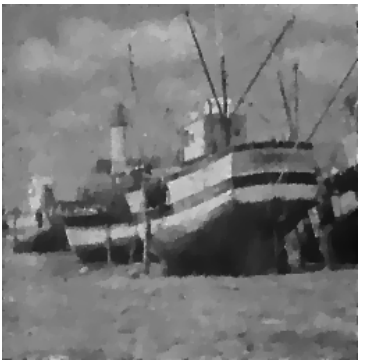

(q)

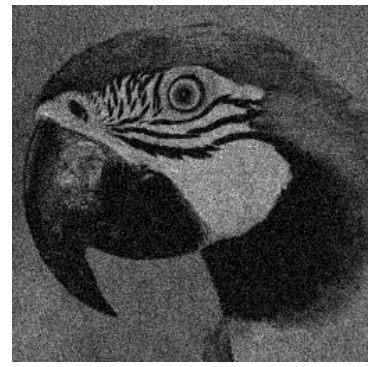

(b)

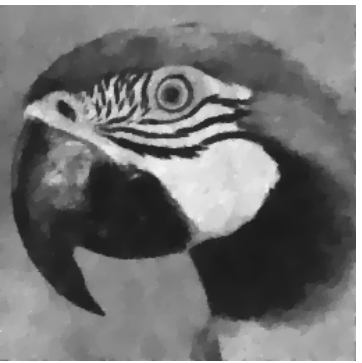

(f)

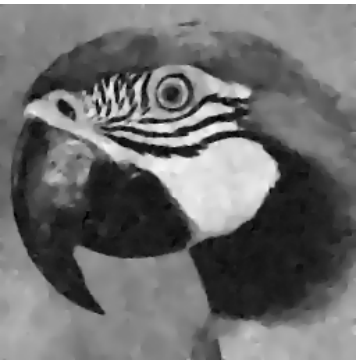

(j)

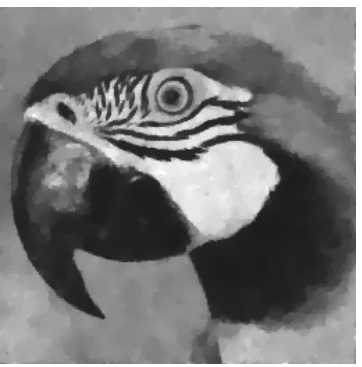

(n)

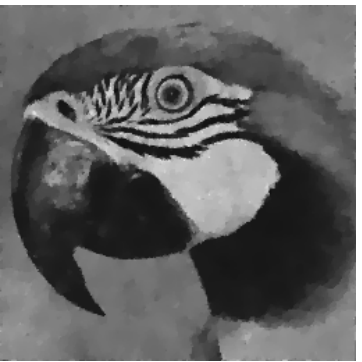

(r)

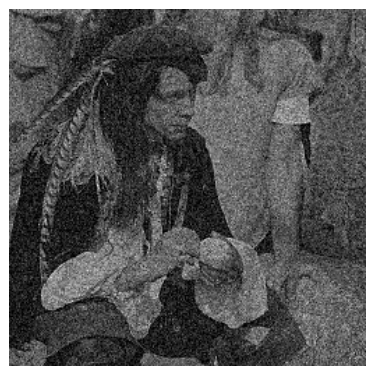

(c)

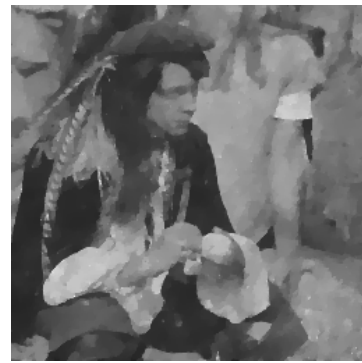

(g)

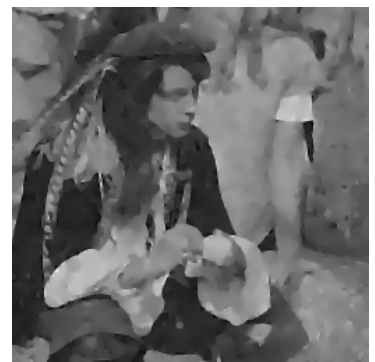

(k)

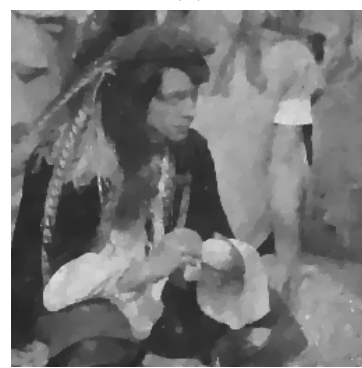

(o)

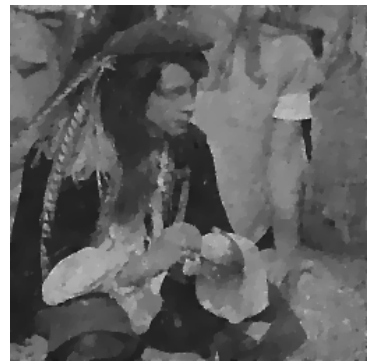

(s)

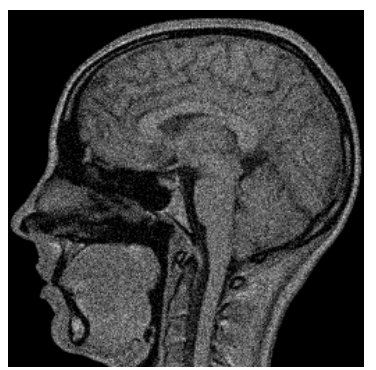

(d)

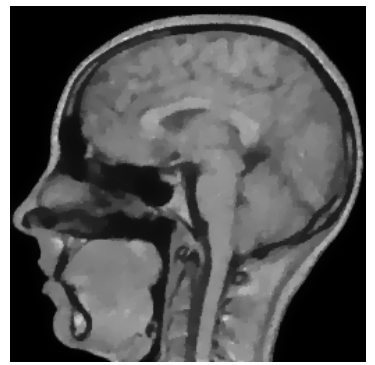

(h)

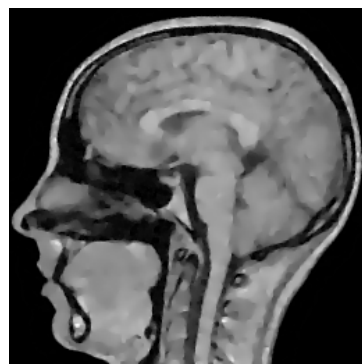

(1)

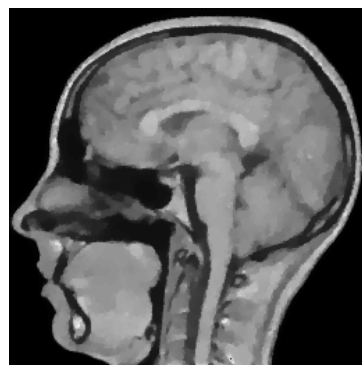

(p)

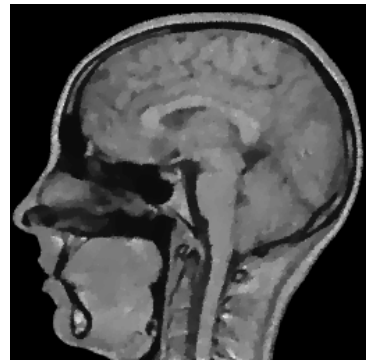

(t)

Figure 4. Recovered results. First row (a - d): Noisy image $f$ with $I_{\max }=90, \sigma=10$; Second row (e - h): restored images using model M1; Third row (i - 1): restored images using model M2; Forth row (m - p): restored images using our model M3 $\gamma=8$ ); Fifth row (q - t): restored images using our model $\alpha-\mathbf{M 3}(\gamma=8)$ 


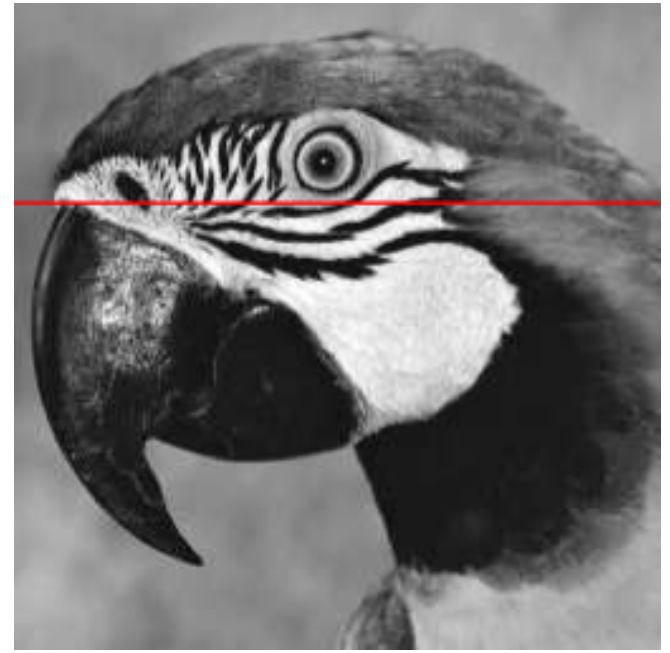

(a)

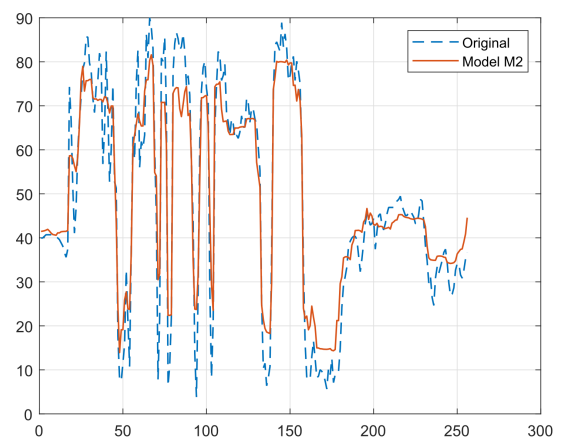

(c)

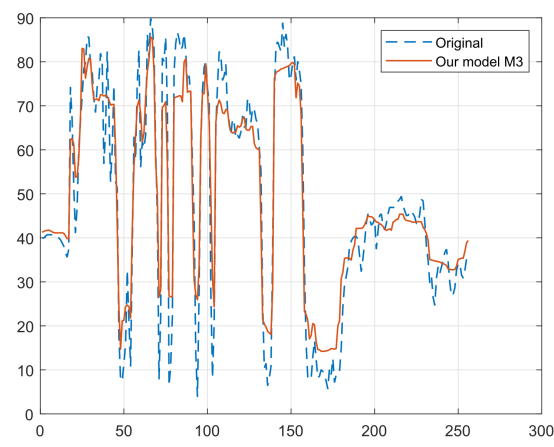

(e)

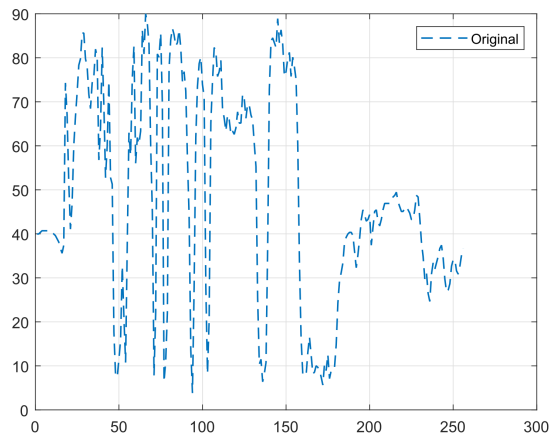

(b)

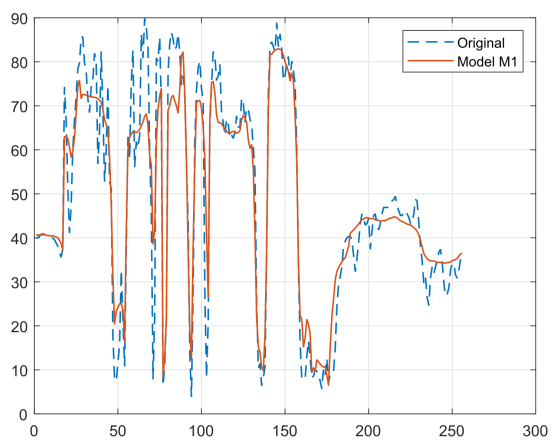

(d)

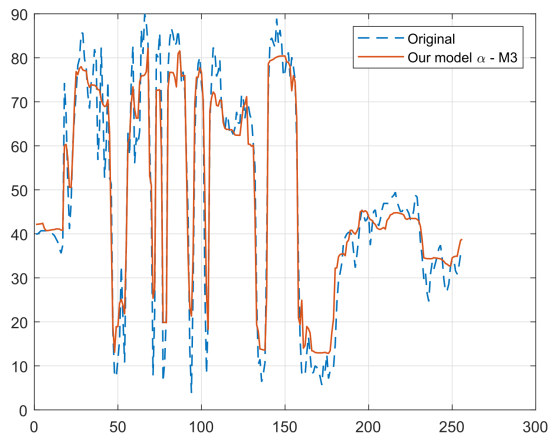

(f)

Figure 5. Image 'parrot'. (a) Original image with the location of the 81th row. 1D line taken across: (b) 81th row of the original image; from (c) to (f): the 81 th row of the restored image from (f), (j), (n) and (r) of the second column in Fig. (4)

linear diffusion. SIAM J. Numer. Anal., 29 (1), pp. 182193.

Chambolle, A. (2004). An algorithm for total variation minimization and applications. J. Math. Imaging Vis., 20 pp. 8997.

De los Reyes, J. C., Schnlieb, C.B. (2013). Image denoising: Learning the noise model via nonsmooth PDE-constrained optimization. Inverse Probl. Imag., 7 (4), pp. 1183-1214.

Deng, W., Yin, W. (2012). On the Global and Linear Convergence of the Generalized Alternating Direc- tion Method of Multipliers. J. Sci. Comput., 66 (3), pp. 889916.

Eckstein, J., Bertsekas, D. (1992). On the DouglasRachford splitting method and the proximal point algorithm for maximal monotone operators. J. Sci. Comput., 55 (1-3), pp. 293318.

Goldstein, T., Osher, S. (2009). The split Bregman method for L1-regularized problems. SIAM J. Imaging Sci., 2 (2), pp. 8997.

Goldstein, T., O’Donoghue, B., Setzer, S., Baraniuk, R. 
Table 2. PSNR values for recovered images given by the compared methods with various levels

\begin{tabular}{|c|c|c|c|c|c|c|}
\hline \multicolumn{2}{|c|}{ Noise levels } & \multicolumn{5}{|c|}{ PSNR } \\
\hline \multicolumn{7}{|c|}{ Boat (256x256) } \\
\hline$I_{\max }$ & $\sigma$ & Noisy & Model 1 & Model 2 & Ours Model 3 & Ours $\alpha$ - model 3 \\
\hline \multirow{2}{*}{120} & 5 & 22.1817 & 26.9722 & 27.4083 & 27.4214 & 27.8203 \\
\hline & 10 & 19.5590 & 25.5219 & 25.8668 & 25.8087 & 26.2691 \\
\hline \multirow{2}{*}{90} & 5 & 20.5147 & 26.2885 & 26.3563 & 26.3608 & 26.8478 \\
\hline & 10 & 17.5246 & 24.8378 & 24.8073 & 24.8023 & 25.1663 \\
\hline \multirow{2}{*}{60} & 5 & 18.0903 & 25.2249 & 24.9089 & 24.9335 & 25.4281 \\
\hline & 10 & 14.5424 & 23.7054 & 23.3545 & 23.3854 & 23.7755 \\
\hline \multicolumn{7}{|c|}{ Parrot (256x256) } \\
\hline \multirow{2}{*}{120} & 5 & 22.6619 & 28.5616 & 28.6885 & 28.6900 & 28.9310 \\
\hline & 10 & 19.8727 & 27.0983 & 27.0003 & 27.0047 & 27.4130 \\
\hline \multirow{2}{*}{90} & 5 & 20.9874 & 27.7751 & 27.6360 & 27.6251 & 27.9465 \\
\hline & 10 & 17.8603 & 26.1740 & 25.7895 & 25.8183 & 26.3000 \\
\hline \multirow{2}{*}{60} & 5 & 18.5235 & 26.5617 & 26.0621 & 26.0553 & 26.6803 \\
\hline & 10 & 14.9155 & 24.2194 & 23.8934 & 23.8581 & 24.5047 \\
\hline \multicolumn{7}{|c|}{ Man (256x256) } \\
\hline \multirow{2}{*}{120} & 5 & 22.6241 & 25.4868 & 25.8171 & 25.8033 & 26.5617 \\
\hline & 10 & 19.7729 & 24.0820 & 24.3303 & 24.3416 & 24.9141 \\
\hline \multirow{2}{*}{90} & 5 & 20.9975 & 24.8372 & 24.7820 & 24.8094 & 25.2636 \\
\hline & 10 & 17.7206 & 23.4761 & 23.4191 & 23.3999 & 23.9755 \\
\hline \multirow{2}{*}{60} & 5 & 18.4461 & 23.7810 & 23.5133 & 23.5145 & 24.0462 \\
\hline & 10 & 14.8019 & 22.4934 & 22.1475 & 22.1458 & 22.6198 \\
\hline \multicolumn{7}{|c|}{ Brain (256x256) } \\
\hline \multirow{2}{*}{120} & 5 & 24.1232 & 28.5295 & 28.5383 & 28.5061 & 29.0337 \\
\hline & 10 & 21.2526 & 26.5321 & 26.4516 & 26.5022 & 27.1849 \\
\hline \multirow{2}{*}{90} & 5 & 22.4320 & 27.3158 & 27.3056 & 27.2517 & 27.9494 \\
\hline & 10 & 19.2605 & 25.1811 & 25.2251 & 25.1725 & 25.9346 \\
\hline \multirow{2}{*}{60} & 5 & 19.9558 & 25.5354 & 25.4818 & 25.4615 & 26.1903 \\
\hline & 10 & 16.2397 & 23.2733 & 23.3101 & 23.2507 & 23.9841 \\
\hline
\end{tabular}

Table 3. SSIM values for recovered images given by the compared methods with various levels

\begin{tabular}{|c|c|c|c|c|c|c|}
\hline \multicolumn{2}{|c|}{ Noise levels } & \multicolumn{5}{|c|}{ SSIM } \\
\hline \multicolumn{7}{|c|}{ Boat (256x256) } \\
\hline$I_{\max }$ & $\sigma$ & Noisy & Model 1 & Model 2 & Ours Model 3 & Ours $\alpha$ - model 3 \\
\hline \multirow{2}{*}{120} & 5 & 0.5142 & 0.7607 & 0.7738 & 0.7719 & 0.7877 \\
\hline & 10 & 0.4038 & 0.6994 & 0.7129 & 0.7107 & 0.7317 \\
\hline \multirow{2}{*}{90} & 5 & 0.4441 & 0.7308 & 0.7316 & 0.7316 & 0.7491 \\
\hline & 10 & 0.3258 & 0.6639 & 0.6655 & 0.6647 & 0.6804 \\
\hline \multirow{2}{*}{60} & 5 & 0.3503 & 0.6805 & 0.6657 & 0.6700 & 0.6914 \\
\hline & 10 & 0.2247 & 0.6055 & 0.5930 & 0.5953 & 0.6126 \\
\hline \multicolumn{7}{|c|}{ Parrot (256x256) } \\
\hline \multirow{2}{*}{120} & 5 & 0.4692 & 0.8434 & 0.8504 & 0.8505 & 0.8544 \\
\hline & 10 & 0.3591 & 0.8150 & 0.8210 & 0.8206 & 0.8247 \\
\hline \multirow{2}{*}{90} & 5 & 0.4058 & 0.8290 & 0.8321 & 0.8328 & 0.8363 \\
\hline & 10 & 0.2957 & 0.7928 & 0.7984 & 0.7976 & 0.8036 \\
\hline \multirow{2}{*}{60} & 5 & 0.3205 & 0.8003 & 0.8045 & 0.8048 & 0.8068 \\
\hline & 10 & 0.2165 & 0.7534 & 0.7587 & 0.7577 & 0.7667 \\
\hline \multicolumn{7}{|c|}{ Man (256x256) } \\
\hline \multirow{2}{*}{120} & 5 & 0.6122 & 0.7202 & 0.7330 & 0.7314 & 0.7637 \\
\hline & 10 & 0.4872 & 0.6421 & 0.6589 & 0.6597 & 0.6893 \\
\hline \multirow{2}{*}{90} & 5 & 0.5440 & 0.6844 & 0.6797 & 0.6798 & 0.6960 \\
\hline & 10 & 0.3994 & 0.6087 & 0.6048 & 0.6055 & 0.6399 \\
\hline \multirow{2}{*}{60} & 5 & 0.4344 & 0.6220 & 0.6051 & 0.6035 & 0.6354 \\
\hline & 10 & 0.2827 & 0.5488 & 0.5260 & 0.5274 & 0.5618 \\
\hline \multicolumn{7}{|c|}{ Brain (256x256) } \\
\hline \multirow{2}{*}{120} & 5 & 0.6823 & 0.8595 & 0.8541 & 0.8572 & 0.8705 \\
\hline & 10 & 0.5897 & 0.8051 & 0.7993 & 0.8037 & 0.8278 \\
\hline \multirow{2}{*}{90} & 5 & 0.6298 & 0.8281 & 0.8177 & 0.8231 & 0.8445 \\
\hline & 10 & 0.5264 & 0.7596 & 0.7478 & 0.7589 & 0.7897 \\
\hline \multirow{2}{*}{60} & 5 & 0.5525 & 0.7726 & 0.7589 & 0.7627 & 0.7931 \\
\hline & 10 & 0.4373 & 0.6860 & 0.6915 & 0.6840 & 0.7166 \\
\hline
\end{tabular}

(2012). Fast alternating direction optimization methods. SIAM J. Imaging Sci., 7 (3), pp. 15881623.

He, C., Hu, C., Zhang, W., Shi, B. (2014). A Fast Adaptive Parameter Estimation for Total Variation Image Restoration. IEEE Trans. Image Process., 23 (12), pp. 4954-4967.
Huang, Y. M. , Ng, M. K., Wen, Y. W. (2008) A fast total variation minimization method for image restoration. Multiscale Model. Sim., 7 (2), pp. 774795.

Le, T., Chartrand, R., Asaki, T.J. (2007). A variational approach to reconstructing images corrupted by Pois- 
son noise. J. Math. Imaging. Vis., 27 pp. 257-263.

Micchelli, C.A., Shen, L., Xu, Y. Proximity algorithms for image models: denoising (2011). Inverse Probl., 27 (4), 045009.

Pham, C. T., Kopylov, A. V. (2015). Multi-quadratic dynamic programming procedure of edge-preserving denoising for medical images. ISPRS - Int. Arch. Photogramm. Remote Sens. Spatial Inf. Sci., XL-5/W6 pp. 101-106.

Pham, C.T., Gamard, G., Kopylov, A., Tran, T.T.T. (2018). An algorithm for image restoration with mixed noise using total variation regularization. Turk. J. Elec. Eng. Comp. Sci., 26 (6), pp. 2831-2845.

Rudin, L., Osher, S., Fatemi, E. (1992). Nonlinear total variation-based noise removal algorithms. Physica $D$, 60 pp. 259-268.

Wang, Y., et al. (2011). MTV: modified total variation model for image noise removal IEEE Electron. Lett., 47 (10), pp. 592-594.

Zhou, W., Lia, Q. (2012). Adaptive total variation regularization based scheme for Poisson noise removal. Math. Method. Appl. Sci., 36 (3), pp. 290299. 